\title{
FORMULATION AND EVALUATION OF ANTIPARKINSON'S DRUG INCORPORATED TRANSDERMAL FILMS
}

\author{
POREDDY SRIKANTH REDDY ${ }^{1 *}$, ALAGARSAMY ${ }^{2}$, SUBHAH CHANDRA BOSE $\mathbf{P}^{1}$, DAMINENI SARITA ${ }^{2}$, SRUTHI V ${ }^{1}$ \\ ${ }^{1}$ Department of Pharmaceutics, MNR College of Pharmacy, Sangareddy, Telangana, India. ${ }^{2}$ Department of Pharmaceutical Chemistry, MNR \\ College of Pharmacy, Sangareddy, Telangana, India. ${ }^{3}$ Department of Pharmaceutics, Sultan-ul-Uloom College of Pharmacy, Hyderabad, \\ Telangana, India. Email: srikanthreddyporeddy@gmail.com
}

Received: 25 July 2019, Revised and Accepted: 08 August 2019

ABSTRACT

Objective: Ropinirole suitable for transdermal delivery due to its small molecular size ( $260.37 \mathrm{~g} / \mathrm{mol}$ ), optimum log p (2.3), and low oral bioavailability $(50 \%)$ due to first-pass metabolism, the short elimination half-life of $4-6 \mathrm{~h}$. Dose of drug is $6 \mathrm{mg} / \mathrm{day}$. Hence, in the present study, an attempt was made to deliver antiparkinson's drug through transdermal route in the form of transdermal film to attain sustained release using different concentration of drug, polymers stabilizers.

Methods: Among the different formulations of matrix type (F1 to F5), F2 and F4 formulations were optimized based on crystallinity. These formulations were carried out for in vitro permeation studies. Out of these two formulations, F4 formulation showed target drug release.

Results and Discussion: The formulation F4 containing 2 mg drug, 600 mg hydroxypropyl methylcellulose E15 and 50 mg of Eudragit RS 100 was selected as optimized formulation, after considering its microscopic examination throughout stability, \% drug content ( $98.4 \%$ ), drug permeated through the cellophane membrane at the end of $72 \mathrm{~h}$, and evaluation of physicochemical characterization parameters such as thickness, weight variation, flatness, folding endurance, moisture content, and tensile strength.

Conclusion: The results of physicochemical characterization were ensured the stability of the films. The drug permeation profile was also found to follow Higuchi kinetic model.

Keywords: Ropinirole, Transdermal film, Permeation studies, Cellophane membrane.

(C) 2019 The Authors. Published by Innovare Academic Sciences Pvt Ltd. This is an open access article under the CC BY license (http://creativecommons. org/licenses/by/4. 0/) DOI: http://dx.doi.org/10.22159/ajpcr.2019.v12i10.35084

\section{INTRODUCTION}

Transdermal drug delivery systems allow delivery of a drug into the systemic circulation through permeation through skin layers at a controlled rate. The skin poses an extremely good barrier to drug penetration, and it is usually necessary to employ enhancement strategies [1,2]. For transdermal products, the goal of dosage design is to maximize the flux through the skin into the systemic circulation and simultaneously minimize the retention and metabolism of the drug in the skin [3]. The drug input can be terminated at any point of time of removing transdermal patches $[4,5]$. The simplified medication regimen leads to improved patient compliance and reduce inter- and intra-patient variables [6]. It is possible that an equivalent therapeutic effect can be elicited through transdermal drug delivery with a lower daily dose than that is required by oral route $[7,8]$.

Ropinirole is used to treat Parkinson's disease drug influences striatal neuronal firing rates through activation of dopamine receptors in the striatum and the Substantia nigra, the site of neurons that send projections to the striatum, improves motor function [9-11]. Ropinirole is a non-ergoline dopamine agonist with high intrinsic activity at the $\mathrm{D}_{2}$ and $\mathrm{D}_{3}$ dopamine receptor subtypes; it binds with higher affinity to $D_{3}$ than to $D_{2}$ or $D_{4}$ receptor subtypes [12]. It is weakly active at the $5-\mathrm{HT}_{2}$, and $\alpha_{2}$ receptors and is said to have virtually no affinity for the 5 - $\mathrm{HT}_{1}$, benzodiazepine, gamma-aminobutyric acid, muscarinic, $\alpha_{1}$, and $\beta$-adrenoreceptors $[13,14]$.

The model drug was chosen as the suitable for formulating a transdermal drug delivery system: Because of the conventional multidose therapy leads to fluctuations in serum levels of drug, and low oral bioavailability due to first-pass metabolism, shorter half-life. The rational strategy to overcome this drawback is to minimize the fluctuations by fabricating sustained-release formulations. Model drug suitable for transdermal delivery due to its molecular size $(260.37 \mathrm{~g} / \mathrm{mol})$, optimum $\log P(2.3)$, and low oral bioavailability $(50 \%)$ due to first-pass metabolism. The short elimination half-life of 4-6 h dose of drug is $6 \mathrm{mg} /$ day. Hence, in the present study, transdermal film for drug was designed and developed to achieve sustained release using different concentration of polymers. METHODS

Ropinirole was procured from Mylan Laboratories Ltd., India. Hydroxypropyl cellulose was procured from Colorcon Asia Pvt., Ltd., India. Eudragit RS 100 was procured from Evonik Pharma, India. Other materials used in the study were of analytical grade.

\section{Preformulation study}

Preformulation involves the application of biopharmaceutical principles to the physicochemical parameters of drug substance is characterized with the goal of designing an optimum drug delivery system. Preformulation studies relate to pharmaceutical and analytical investigation carried out proceedings and supporting formulation development efforts of the dosage forms of the drug substance. Preformulation yields basic knowledge necessary to develop suitable formulation. It gives information needed to define the nature of the drug substance and provide framework for drug combination with polymers in the dosage form. Hence, the following preformulation studies were carried out $[15,16]$

1. Identification of drug

- Standard calibration curve

- Solubility analysis.

2. Partition coefficient. 


\section{Identification of drug}

Determination of $\lambda_{\max }: 25 \mathrm{mg}$ of pure drug was taken and known concentration of drug solution was prepared by diluting drug solution in methanol. The solutions were scanned from 200 to 400 $\mathrm{nm}$ against the reagent blank to fix absorption maxima. Spectrum of the model drug was obtained and $\lambda_{\max }$ of model drug was found to 249 $\mathrm{nm}$. Hence, all further investigations were carried out at the same wavelength.

\section{Standard calibration curve}

Standard stock solution

Transfer an accurately weighed amount of about $25 \mathrm{mg}$ of drug and working standard into a $50 \mathrm{ml}$ volumetric flask. Dissolve and make up the volume with methanol [17]

\section{Standard preparation}

Pipette $5 \mathrm{ml}$ of the standard stock solution into a $50 \mathrm{ml}$ volumetric flask, make up the volume with methanol.

Solution: (a) Std. stock solution $500 \mu \mathrm{g} / \mathrm{ml}$ in methanol and (b) working stock $50 \mu \mathrm{g} / \mathrm{ml}$ in methanol.

From the stock solution, different aliquots were taken in series of 10 $\mathrm{ml}$ volumetric flasks and volume made up with buffer to get a series of working standard solutions of concentrations, $5 \mu \mathrm{g} / \mathrm{ml}, 10 \mu \mathrm{g} / \mathrm{ml}$, $15 \mu \mathrm{g} / \mathrm{ml}, 20 \mu \mathrm{g} / \mathrm{ml}$, and $25 \mu \mathrm{g} / \mathrm{ml}$. The absorbance of samples was obtained spectrophotometrically against the reagent blank at $249 \mathrm{~nm}$. The calibration curves were constructed by plotting drug concentration versus the absorbance value at $249 \mathrm{~nm}$ and the regression equation was computed.

\section{Solubility studies}

The solubility of drug is an important physicochemical property because it affects the bioavailability of the drug, and hence the therapeutic efficiency of the pharmaceutical product. The solubility of the molecules in various solvents is determined as a first step. This information is valuable in developing a formulation. Solubility was determined in organic solvents [18].

\section{Solubility study of model drug in different media}

Solubility studies were performed by taking required quantity of drug in $1 \mathrm{ml}$ of different organic solvents (methanol, ethanol, ethyl acetate, isopropyl alcohol, heptane, and water) separately up to its saturation and subjected to mechanical shaking at $100 \mathrm{rpm}$ for $24 \mathrm{~h}$. The resultant dispersions were collected and subjected to centrifugation and the concentration of drug was determined from absorbance at $249 \mathrm{~nm}$.

\section{Partition coefficient}

The partition coefficient is the ratio of concentrations of a compound in the two phases of a mixture of two immiscible solvents at equilibrium. Normally, one of the solvents chosen is phosphate buffer ( $\mathrm{pH}$ 7.4), while the second is hydrophobic such as octanol. Standard solution of drug was prepared in phosphate buffer ( $\mathrm{pH}$ 7.4). The classical and most reliable method of log p determination is the shake-flask method, which consists of dissolving some of the solute in question in a volume of octanol and phosphate buffer ( $\mathrm{pH}$ 7.4), then measuring the concentration of the solute in each solvent [19].

\section{Experimental method}

\section{Preparation of ropinirole transdermal film}

Transdermal film containing ropinirole was prepared by the solvent casting technique using aluminum foil as a backing layer. The two independent factors were hydroxypropyl methylcellulose E15 and Eudragit RS 100. The mixture of solvent casting method consisted of solvent (Ethanol), permeation enhancer (Tween 80, 5\% of total weight of drug-polymer), and plasticizer (polyethylene glycol, $2.5 \%$ of total volume of solvent). Dose of drug was adjusted in such a way that square patch $(2 \mathrm{sq} . \mathrm{cm})$ consisted of $2 \mathrm{mg}$ of ropinirole. The films were stored between sheets of wax paper in a desiccator. The formulation chart of transdermal films is depicted in Table 1.

\section{Physicochemical characterization of films \\ Drug content}

Films of a specified area $\left(1 \mathrm{~cm}^{2}\right)$ were dissolved in $5 \mathrm{~mL}$ of dichloromethane, and the volume was made up to $10 \mathrm{~mL}$ with phosphate buffer $\mathrm{pH}$ 7.4, dichloromethane was evaporated using a rotary vacuum evaporator at $45^{\circ} \mathrm{C}$. A blank was prepared using a drug-free film treated similarly. The solutions were filtered through a $0.45 \mu \mathrm{m}$ membrane, diluted suitably and absorbance were read at $249 \mathrm{~nm}$ in a double beam ultraviolet (UV)-visible spectrophotometer.

\section{Thickness}

The thickness of films was measured at three different places using a digital micrometer (Mitutoyo Co., Japan) and mean values were calculated [20]

\section{Weight variation}

The films were subjected to mass variation by individually weighing randomly selected films. Such determinations were carried out for each formulation [20].

\section{Flatness}

Three longitudinal strips were cut out from each film: One from the center, one from the left side, and one from the right side. The length of each strip was measured and the variation in length because of non-uniformity in flatness was measured by determining percent constriction, with $0 \%$ constriction equivalent to $100 \%$ flatness [21]

\section{Folding endurance}

Folding endurance was determined by repeatedly folding one film at the same place until it broke. The number of times the film could be folded at the same place without breaking/cracking gave the value of folding endurance [22]

\section{Tensile strength}

To determine the elongation as a tensile strength, the polymeric film was pulled by means of a pulley system; weights were gradually added to the pan to increase the pulling force until the film was broken [21]. The elongation, i.e., the distance traveled by the pointer before break of the film was noted with the help of magnifying glass on the graph paper, the tensile strength was calculated as $\mathrm{kg}-\mathrm{cm}^{-2}$.

\section{Percentage of moisture content}

The films were weighed individually and kept in a desiccators containing activated silica at room temperature for $24 \mathrm{~h}$. Individual films were weighed repeatedly until they showed a constant weight. The percentage of moisture content was calculated as the difference between initial and final weight with respect to final weight [23]

\section{In vitro skin permeation studies}

In vitro skin permeation studies were performed using a Franz diffusion cell with a receptor compartment capacity of $22.5 \mathrm{~mL}$. The excised rat abdominal skin (Wistar albino) was mounted between the donor and receptor compartment of the diffusion cell. The formulated

\section{Table 1: Formulation chart of transdermal films}

\begin{tabular}{llllll}
\hline Ingredients & F1 & F2 & F3 & F4 & F5 \\
\hline Ropinirole base & 2 & 2 & 2 & 2 & 2 \\
hydroxypropyl methylcellulose E15 (mg) & 100 & 150 & 200 & 250 & 50 \\
Eudragit RS100 (mg) & 200 & 150 & 100 & 50 & 250 \\
Tween 80 (\%w/w) & 5 & 5 & 5 & 5 & 5 \\
Polyethylene glycol (\%w/w) & 2.5 & 2.5 & 2.5 & 2.5 & 2.5 \\
Ethanol (ml) & 2.5 & 2.5 & 2.5 & 2.5 & 2.5 \\
\hline
\end{tabular}


films were placed over the skin and covered with paraffin film. The receptor compartment of the diffusion cell was filled with phosphate buffer $\mathrm{pH}$ 7.4. The whole assembly was fixed on a magnetic stirrer, and the solution in the receptor compartment was constantly and continuously stirred using magnetic beads at $50 \mathrm{rpm}$; the temperature was maintained at $32 \pm 0.5^{\circ} \mathrm{C}$. The samples were withdrawn at different time intervals and analyzed for drug content spectrophotometrically. The receptor phase was replenished with an equal volume of phosphate buffer $\mathrm{pH} 7.4$ at each sample withdrawal. The cumulative percentages of drug permeated per square centimeter of films were plotted against time $[24,25]$.

Kinetic modeling of drug release

To analyze the mechanism of drug release from the films, the release data were fitted to the following equations [26]:

Zero-order equation:

$$
Q=k_{0} t
$$

Where $Q$ is the amount of drug released at time $t$, and $k_{0}$ is the release rate.

First-order equation:

$$
\ln (100-Q)=1 \text { n } 100-k_{1} t
$$

Where $Q$ is the percent of drug release at time $t$, and $k_{1}$ is the release rate constant.

Higuchi's equation:

$$
Q=k 2 \sqrt{ } t
$$

Where $Q$ is the percent of drug release at time $t$, and $k_{2}$ is the diffusion rate constant.

\section{RESULTS AN DISCUSSION}

\section{Identification of drug}

\section{Calibration curve}

Determination of $\lambda$ max: UV-spectra of ropinirole are shown in Fig. 1. The linear equation in methanol was $\mathrm{y}=0.0416 \times$ concentration $(\mathrm{mcg} / \mathrm{ml})$. Different standard concentration and their absorbance values were shown. At all the concentration levels, the standard deviation was low. Goodness-of-fit of regression equation was supported by high regression value (0.9998). Hence, the developed method can be used for routine analysis of the drug in pharmaceutical formulations studies. Calibration curve data of the ropinirole in methanol are depicted in Fig. 2.

\section{Solubility analysis}

Solubility studies showed that the ropinirole shows organic solubility. It showed maximum solubility in ethanol, methanol, and minimum solubility in isopropyl alcohol and water. The comparison solubility profile of ropinirole in different solvents is depicted in Fig. 3.

\section{Partition coefficient}

In the present study, $\mathrm{pH} 7.4$ phosphate buffer was used as in vitro study fluid and the solubility drug in $\mathrm{pH} 7.4$ buffer was found to be $0.456 \mathrm{mcg} / \mathrm{ml}$. The logarithmic value of the partition coefficient (log p) was found to be 2.1. The results indicated that the drug has optimum $\log \mathrm{p}$ value, which fulfilled the requirements of formulating it into a transdermal patch. The calibration curve of model drug in $\mathrm{pH} 7.4$ buffer is shown in Fig. 4.

Physicochemical characterization of films

Drug content (Assay): The drug content of different formulations (F1-F5) was ranged between 93.2 and $98.4 \%$. The drug content was nearly the same as the dose of the drug in all the patches. The cumulative percentage drug permeated and percentage drug retained by individual patch in in vitro permeation studies were based on the mean amount of drug present in the respective patch. The drug content of different formulations (F1-F5) is shown in Table 2 .

Table 2: Drug content (Assay)

\begin{tabular}{lll}
\hline S. No. & Formulation code & Drug content \% \\
\hline 1 & F1 & 95.8 \\
2 & F2 & 93.2 \\
3 & F3 & 96.9 \\
4 & F4 & 98.4 \\
5 & F5 & 94.1 \\
\hline
\end{tabular}

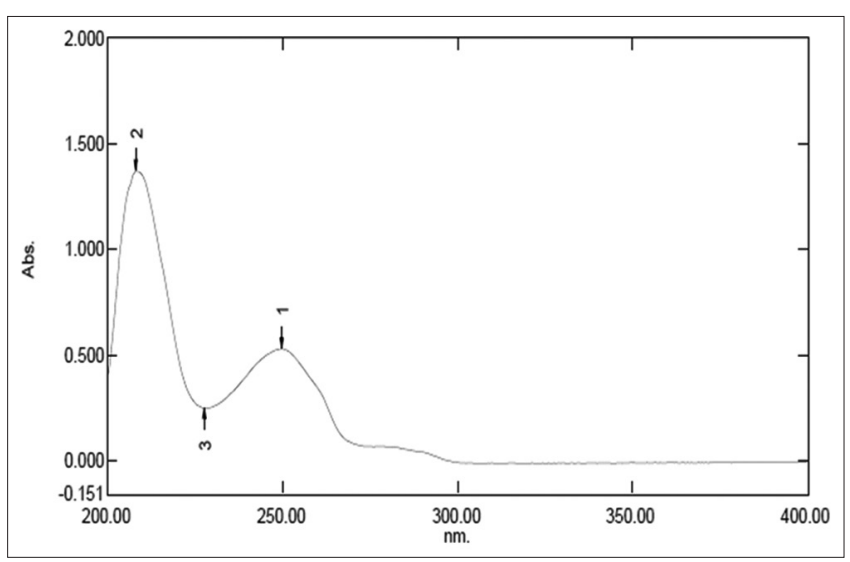

Fig. 1: Ultraviolet -spectra of drug

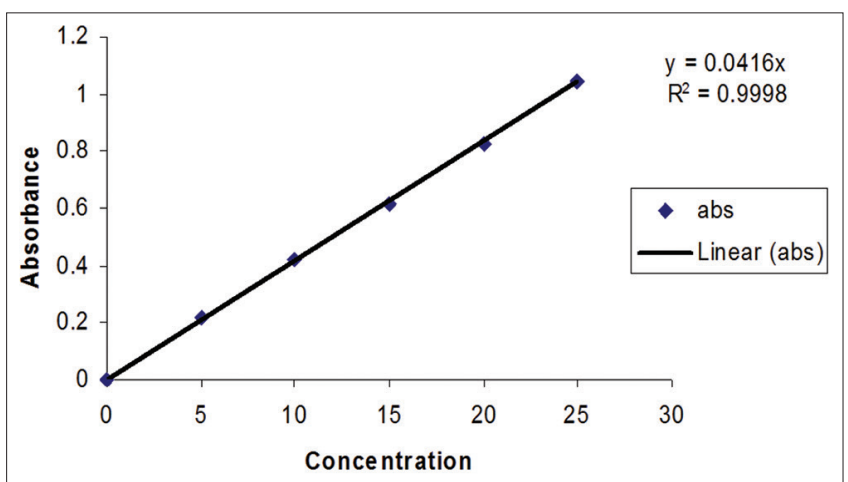

Fig. 2: Calibration curve data of the atmospheric pressure ionization (ropinirole) in methanol

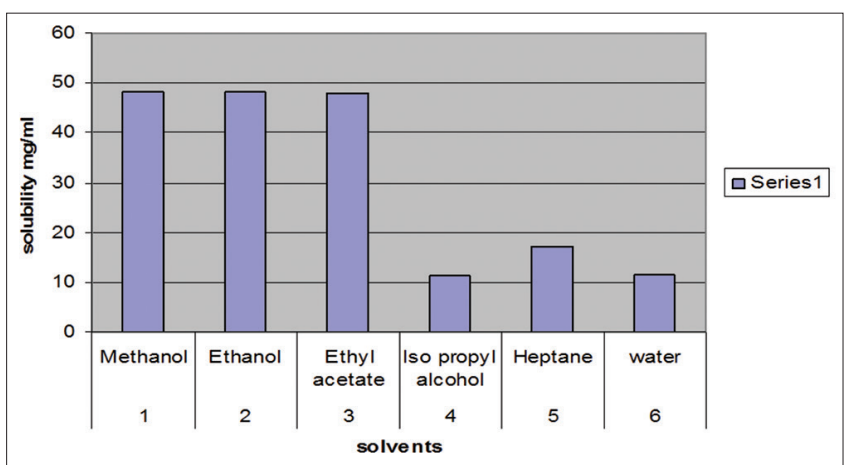

Fig. 3: Comparison of solubility profile of atmospheric pressure ionization in different solvents 
Table 3: Physicochemical characterization of prepared transdermal films

\begin{tabular}{|c|c|c|c|c|c|}
\hline Formulation code & Thickness $(\mu \mathrm{m})$ & Weight variation $\left(\mathrm{mg} \mathrm{cm}^{-2}\right)$ & Folding endurance & Tensile strength $\left(\mathrm{kg} \mathrm{cm}^{-2}\right)$ & Moisture content (\%) \\
\hline $\mathrm{F} 1$ & $112 \pm 2.4$ & $13.58 \pm 0.62$ & $76.21 \pm 1.08$ & $3.82 \pm 0.068$ & $2.73 \pm 0.44$ \\
\hline $\mathrm{F} 2$ & $127 \pm 6.5$ & $12.46 \pm 0.39$ & $69.76 \pm 1.12$ & $2.65 \pm 0.093$ & $1.95 \pm 0.85$ \\
\hline F3 & $141 \pm 7.3$ & $15.19 \pm 0.32$ & $82.94 \pm 2.54$ & $2.99 \pm 0.064$ & $2.81 \pm 0.14$ \\
\hline $\mathrm{F} 4$ & $133 \pm 1.8$ & $12.25 \pm 0.87$ & $88.56 \pm 1.96$ & $3.86 \pm 0.019$ & $2.84 \pm 0.58$ \\
\hline F5 & $140 \pm 3.4$ & $14.26 \pm 0.54$ & $74.35 \pm 1.89$ & $3.07 \pm 0.094$ & $1.94 \pm 0.69$ \\
\hline
\end{tabular}

Table 4: Regression coefficient values of different kinetic models

\begin{tabular}{lllll}
\hline Formulation code & Higuchi kinetics $\left(\mathbf{R}^{2}\right)$ & Peppas plot $\left(\mathbf{R}^{2}\right)$ & Zero-order kinetics $\left(\mathbf{R}^{2}\right)$ & First-order kinetics $\left(\mathbf{R}^{2}\right)$ \\
\hline F1 & 0.9894 & 0.9124 & 0.9014 & 0.9129 \\
F2 & 0.9865 & 0.9308 & 0.9333 & 0.9308 \\
F3 & 0.9834 & 0.9266 & 0.9246 & 0.9266 \\
F4 & 0.9877 & 0.9126 & 0.9059 & 0.9126 \\
F5 & 0.9926 & 0.9515 & 0.9536 & 0.9515 \\
\hline
\end{tabular}

The results of the physicochemical characterization, i.e., thickness, weight variation, folding endurance, tensile strength, and moisture content of the films are shown in Table 3. The thickness ranged between $112 \pm 2.4$ and $141 \pm 7.3 \mu \mathrm{m}$, which indicated that the prepared films were uniform in thickness. The weights ranged between $12.25 \pm 0.87 \mathrm{mg}$ and $15.19 \pm 0.32 \mathrm{mg}$, which indicates that different batches film weights were relatively similar. The results indicated that the process employed to prepare transdermal films in this study was capable of producing films with uniform drug content and minimal film variability. The flatness study showed that all the prepared film formulations had the same strip length before and after their cuts, indicated $100 \%$ flatness. Thus, no amount of constriction was observed; all films had a smooth, flat surface and that smooth surface could be maintained when the film was applied to the skin. Folding endurance test results indicated that the films would not break and would maintain their integrity with general skin folding when applied. The moisture content of the prepared formulations was low, which could help the formulations remain stable and reduce brittleness during long-term storage.

\section{In vitro skin permeation studies}

In the in vitro drug permeation studies, the cumulative amount of drug permeated per sq. $\mathrm{cm}$ from different formulations having different concentrations of drug and polymers showed variable permeation patterns. The process of drug permeated in most of the controlled/ sustained release devices including transdermal patches is governed by diffusion. The mean cumulative amounts of drug permeated (In vitro permeation) from different concentrations up to $72 \mathrm{~h}$ were analyzed. Out of all these different formulations F4 formulation showed targeted drug permeation. Studies revealed that the maximum drug permeation was found (97.5\%), as the concentration of drug increases, amount of drug permeated also increases. The in vitro permeation studies data have shown that drug release from the patch formulation has been affected by types of polymer and concentration of polymer, concentration of drug. The in vitro drug permeation profile of cumulative amount permeated formulations (F1-F5) is depicted in Fig. 5.

\section{Kinetic modeling of drug release}

To analyze the mechanism of drug release from the films, the release data were fitted to the various kinetic models, i.e., Higuchi model, Peppas plot, zero-order, and first-order kinetics. The obtained results are shown in Table 4. From the results, Higuchi kinetic model was showed good regression coefficient $\left(\mathrm{R}^{2}\right)$ for all prepared formulations.

\section{CONCLUSION}

In the present research, ropinirole transdermal films were prepared by solvent casting technique using different polymer ratio. All film formulations were checked for physicochemical properties such as thickness, weight variation, and drug content, flatness, folding endurance, and moisture content and showed good results. The

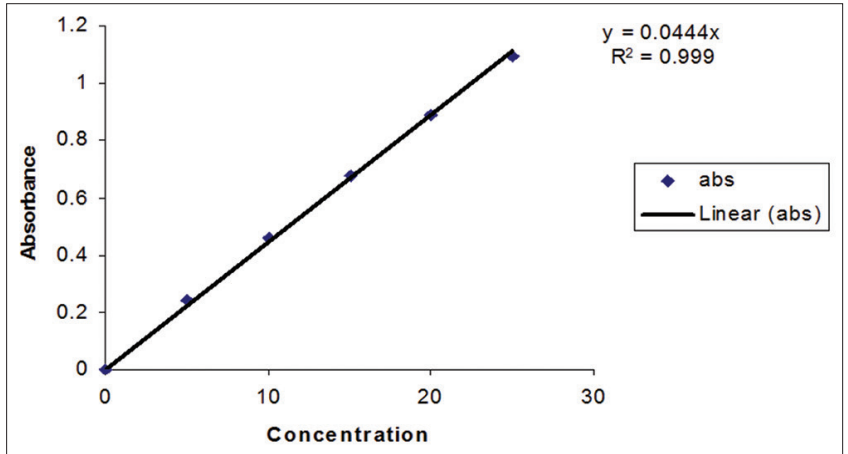

Fig. 4: Calibration curve of model drug in pH 7.4 buffer

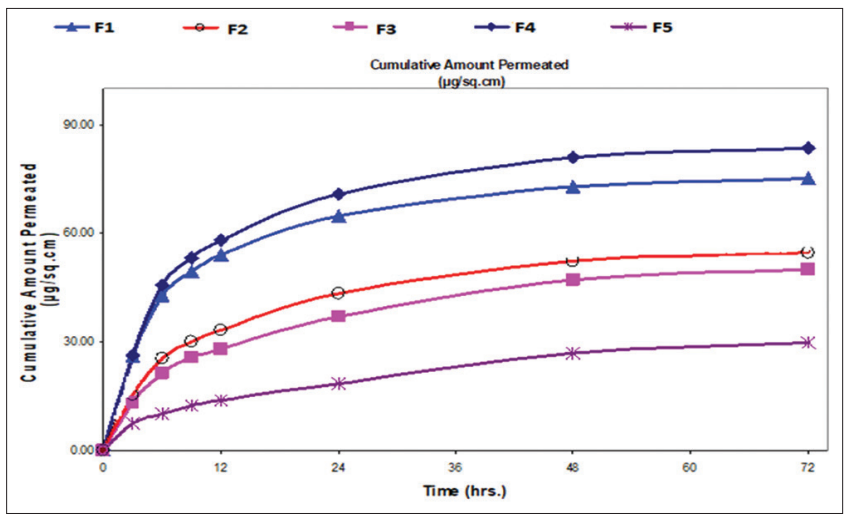

Fig. 5: In vitro drug permeation profile of cumulative amount permeated formulations

in vitro drug permeation data showed that drug release from the films formulations has been affected by types of polymer and concentration of polymer. Drug release data were fitted to various kinetic models, and Higuchi model showed good regression coefficient. The finding of this result revealed that the problems of ropinirole on oral administration such as dissolution rate-limited absorption and gastric side effects can be overcome by applying ropinirole topically in the form of transdermal film.

\section{AUTHORS' CONTRIBUTIONS}

All authors contributed equally to the paper.

\section{CONFLICTS OF INTEREST}

The authors have none to declare 


\section{REFERENCES}

1. Barry B. Transdermal drug delivery. In Aulton ME, editor. Pharmaceutics: The Science of Dosage Form Design. New York: Churchill Livingston; 2002. p. 499-533.

2. Eseldin K, Rakesh KS. Transdermal drug delivery system. Design and evaluation. Int J Adv Pharm Sci 2010;1:201-11.

3. Sahu RK. Development and evaluation of transdermal patches of colchicines. Scho Res Lib 2011;4:330-43.

4. Meenakshi B. Devlopment and characterization of transdermal patches of metoprolol tartrate. Asian J Pharm Clin Res 2010;3:130.

5. Gordon RD, Peterson TA. About transdermal drug delivery. Drug Del Tech 2003;3:44-50.

6. Bhosale N. Formulation and evaluation of transdermal patches of ropinirole HCl. Res J Pharm Biol Chem Sci 2011;2:138

7. Reichmann H. Transdermal delivery of dopamine receptor agonists. Parkinsonism Relat Disord 2009;4:93-6.

8. Vijayan V. Development and physiochemical, in vitro evaluation of antihypertensive transdermal patches. J Pharm Sci Res 2010;2:171-7.

9. Curran MP, Perry CM. Cabergoline: A review of its use in the treatment of parkinson's disease. Drugs 2004;64:2125-41.

10. Reynolds NA, Wellington K, Easthope SE. Rotigotine: In parkinson's disease. CNS Drugs 2005; 19:973-81.

11. Kaestli LZ, Wasilewski-Rasca AF, Bonnabry P, Vogt-Ferrier N. Use of transdermal drug formulations in the elderly. Drugs Aging 2008;25:269-80.

12. Nashatizadeh MM, Lyons KE, Pahwa R. A review of ropinirole prolonged release in parkinson's disease. Clin Interv Aging 2009;4:179-86.

13. Chandrashekar NS, Shobha Rani RH. Physicochemical and pharmacokinetic parameters in drug selection and loading for transdermal drug delivery. Indian J Pharm Sci 2008;70:94-6.

14. Bhosale NR, Hardikar SR, Bhosale AV. Formulation and evaluation of transdermal patches of ropinirole. HCl. Res R Pharm Biol Chem Sci $2011 ; 2: 138-45$.
15. Shivaraj A. Design and evaluation of transdermal drug delivery of ketotifen fumarate internatio. Int J Pharm Biomed Res 2010;1:42-7.

16. Dangi A. Formulation and evaluation of transdermal ondansetron hydrochloride matrix patch: In vitro skin permeation and irritation study. Int J Pharm Res Allied Sci 2012;1:26-34.

17. Sanjay D, Ananya M. Preparation of carvedilol transdermal patch and the effect of propylene glycol on permeation. Int J Pharm Pharm Sci 2010;2:83.

18. Ganju E. Formulation and evaluation of transdermal patch of prochlorperazine maleate for hyperemesis gravidarum. Int J Res Pharm Chem 2011;1:1115-8.

19. Raju RT. Formulation and evaluation of transdermal drug delivery systems for lercanidipine hydrochloride. Int $\mathrm{J}$ Cur Pharm Res 2010;2:253-8.

20. Arora P, Mukherjee B. Design, development, physicochemical, and in vitro and in vivo evaluation of transdermal patches containing diclofenac diethylammonium salt. J Pharm Sci 2002;91:2076-89.

21. Amnuaikit C, Ikeuchi I, Ogawara K, Higaki K, Kimura T. Skin permeation of propranolol from polymeric film containing terpene enhancers for transdermal use. Int J Pharm 2005;289:167-78.

22. Kusum Devi V, Saisivam S, Maria GR, Deepti PU. Design and evaluation of matrix diffusion controlled transdermal patches of verapamil hydrochloride. Drug Dev Ind Pharm 2003;29:495-503.

23. Gupta R, Mukherjee B. Development and in vitro evaluation of diltiazem hydrochloride transdermal patches based on povidoneethylcellulose matrices. Drug Dev Ind Pharm 2003;29:1-7.

24. Dipanjana AS, Sutapa BM, Gopa RB. Oleogels of olive oil and soybean oil for topical drug delivery: A comparative analysis. Int J Pharm Pharm 2019;11:4-10

25. Dina MH, Nermin MS, Vivian SE, Wedad S. Enhancing the sunscreen efficacy of bemotrizinol micropigment by using o/w nanoemulsion topical preparations. Int J Pharm Pharm Sci 2019;11:47-56.

26. Rakesh PP, Grishma P, Ashok B. Formulation and evaluation of transdermal patch of aceclofenac. Int J Drug Del 2009;1:41-51. 